\title{
Review of: "Mechanochemical transformation of planar polyarenes to curved fused-ring systems"
}

\author{
László Jicsinszky
}

Potential competing interests: The author(s) declared that no potential competing interests exist.

The manuscript by Yong and coworkers is encouraging, albeit the synthetic application of ball milling to the aromatic nucleophilic substitution is not new. The usefulness of the target compound is also quite questionable. The content is appropriate to the journal, the descriptions of the compounds are correct, and the authors provide a comprehensive discussion of possible reaction mechanisms.

Among some minor issues, the significant deficiency of the manuscript is the missing scaled-up synthesis at least to gram level. The authors themselves stated the easy reaction scalability but omitted its verification. The mg-scale reaction is not a proven preparative method for a compound that has a $\mathrm{kg}$-scale production process.

1. The authors use a vibrating ball mill in their syntheses which significantly limits batch sizes of the reaction. Besides this limiting factor, the planetary version of the ball mill not only allows the preparative work on multigram-/eventually kg-scale syntheses, but many times, the reactions can be faster, and the scaling-up is easier. The reviewer understands the limited instrumentation, but the current work does not allow the authors to draw the intended general conclusions.

2. The physical description of the Compound 3 is missing. Is that oil, amorphous solid, or possibly crystalline?

3. Usually, the yields are impressive, but the ten mg-scale reactions are rather test-tube reactions or proof of concept experiments than preparative works. Consequently, the presented yields of Compound 4, 8, and corannulene from Compound 1 appear to be excessive. Yields of syntheses on a couple of ten mg-scale, or even around one hundred mg-scale, is more a desire than a fact and does not have meaning or relevances for real production intentions. The proof of the last sentence above the Result and Discussion section ("It is also a practically simple, mild, fast, high-yielding and a sustainable synthetic approach.") is missing. Additionally, in the ESI, the impurities in NMR spectra suggest that the calculated yields on the mg scale are exaggerating.

4. The authors' concern about the carbon tetrachloride toxicity is interesting because all other reagents used in the syntheses can provide more worrying, including the target molecule and intermediates themselves, than $\mathrm{CCl} 4$.

5. In Fig. 5, the authors compared the kg-scale synthesis yield with the mg-scale version. This operation is like comparing the production of a single stalk of wheat with that of a grain field. It is a particular weakness regarding the yield calculation of impure compounds $(1,4,8)$. 
6. ESI requires a Table of Contents. 